Commun. Fac. Sci. Univ. Ank, Series Al

V. 45. pp. 61-66 (1996)

\title{
ON A LAGUERRE INVERSION
}

EMINE SOYTÜRK* and RÜSTEM KAYA**

* Department of Mathematics, University of Ankara, Ankara-TURKEY

** Department of Mathematics, University of Osmangazi, Eskişehir-TURKEY

(Received Nov. 16, 1995; Revised April 15, 1996; Accepted July 30, 1996)

\section{ABSTRACT}

The existence of some automorphisms on certain Laguerre plane L is used to determine the algebraic structure of the projective plane, which is a completion of the affine plane $\mathrm{L}_{\mathrm{A}}$ derived at a known point $\mathrm{A}$. In this paper, firstly a special Laguerre automorphism, that is a Laguerre inversion, is defined; and then the set of fixed points and the set of fixed circles of the Laguerre inversion are investigated.

\section{INTRODUCTION}

A Laguerre plane is a system $(\mathcal{P}, \mathcal{C}, \in)$ which consists of a nonempty set $\mathcal{P}$ of points, a nonempty set $\mathcal{C}$ of subsets (circles) of $\mathcal{P}$ and $\in$ the set theoretical inclusion satisfying the following axioms [2], [4]:

L.1. For every three pairwise nonparallel points $A, B, C$, there is a unique circle containing them. This circle is denoted by $(A B C)$.

(Two points $A, B \in \mathcal{P}$ are said to be parallel if and only if $A=B$ or there is no such a circle $\subsetneq$ that $A, B \in c$. This relation is an equivalance relation on the point set $\mathcal{P}$. Its equivalance classes are called generators. If $\mathrm{A}$ and $\mathrm{B}$ are parallel points, then we write $\mathrm{A} / \mathrm{B}$.)

L.2. For every point there exists a unique generator, containing this point.

L.3. Every circle intersects every generator, containing this point.

L.3. Every circle intersects every generator in exactly one point.

L.4. For each circle $\varsigma$, each point $A$ on $\varsigma$, and each point $B H A$ in $\mathrm{L} \backslash \mathrm{c}$, there is a unique circle $\mathcal{\xi}^{\prime}$ containing $\mathrm{A}$ and $\mathrm{B}$ such that

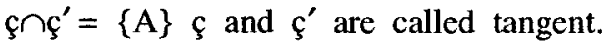


L.5. Every circle $̧$ contains at least three points and $L / c ̧ \neq \varnothing$.

The origin of the Laguerre geometry is the geometry of oriented lines and oriented circles with nonnegative radius of the Euclidean plane [2]. In [1], Benz constructed the following class of Laguerre planes. Let $F$ be an arbitrary field and $\mathrm{V}=\mathrm{F}^{3}$ denote the three dimension vector space over $\mathrm{F}$.

Let $O$ be an oval in the planc $\{(x, y, z) \in V: z=0\}$, i.e., is a subset of a projective plane such that i) each line cuts $O$ in at most two points, and ii) through each point $\mathcal{P} \in \mathcal{O}$ there exists exactly one tangent, i.e. a line intersecting $\mathcal{O}$ in exactly one point. Then $(\mathcal{P}, \mathcal{C}, \epsilon)$ with $\mathcal{P}=\{(\mathrm{x}, \mathrm{y}, \mathrm{z}) \in \mathrm{V}$ : $(\mathrm{x}, \mathrm{y}, 0) \in \mathcal{O}\}$ and $\mathcal{C}=\{(\mathrm{x}, \mathrm{y}, \mathrm{z}) \in \mathcal{P}: \mathrm{z}=\mathrm{ax}+\mathrm{by}+\mathrm{c}, \mathrm{a}, \mathrm{b}, \mathrm{c} \in \mathrm{F}\}$ is a Laguerre plane in the above sense. Here $P$ is the set of points of an ovoidal cylinder and a circle is intersection of $\mathcal{P}$ with a plane which is not parallel to the axis of the cylinder (For a general definition, see [4]). In [2], Benz shows that if 0 is given by $x^{2}+y^{2}=1$ then the corresponding Laguerre plane is isomorphic to the parabolic model $(\mathcal{P}, \mathcal{C}, \in)$ over $R$ where $P=R^{2} \cup R$;

$$
\mathcal{C}=\left\{\left\{(\mathrm{x}, \mathrm{y}) \in \mathbb{R}^{2}: \mathrm{y}=\mathrm{ax} \mathrm{x}^{2}+\mathrm{bx}+\mathrm{c}\right\} \cup\{\mathrm{a}\}: \mathrm{a}, \mathrm{b}, \mathrm{c}, \in R\right\}
$$

and $R$ is the set of real numbers. In order to obtain some new Laguerre planes, Hartman replaced the parabolas in the above model by some particularly chosen curves [3].

The following theorem explains the main property of the Laguerre planes.

Theorem 1. Let $\bar{X}$ be the generator on the point $X$. Then the geometrical structure,

$$
\left.\mathrm{L}_{\mathrm{A}}=(\mathcal{P} \overline{\mathrm{A}},\{\mathcal{f} \backslash\{\mathrm{A}\}: \mathrm{A} \in \boldsymbol{c}, \hat{\in} \in \mathcal{C}\} \cup \overline{\mathrm{X}}: \mathrm{X} \in \mathcal{P}, \mathrm{X} H \mathrm{~A}\}, \in\right)
$$

is an affine plane.

This affine plane is called the affine plane derived by the point $A$ of the Laguerre plane, [2].

\section{HYPERBOLIC AND PARABOLIC PENCILS}

The set of all circles containing nonparallel points $A$ and $B$ is called a hyperbolic pencil and denoted by 


$$
[\mathrm{A}, \mathrm{B}]=\{c: \mathrm{A}, \mathrm{B} \in \boldsymbol{c}, \boldsymbol{\epsilon} \in \mathcal{\varphi}\}
$$

The set of all circles, which are tangent to the circle $\xi_{\text {at }}$ at the given point $A$ is called a parabolic pencil and denoted by $[A, c]$,

$$
[A, c ̧]=\left\{\varsigma^{\prime}: \mathfrak{c}^{\prime} \in \mathcal{C}, \varsigma^{\prime} \cap \mathfrak{s}=\{A\}\right\} \cup\{\varsigma\},
$$

Definition 1. Let $\mathrm{L}=(\mathcal{P}, \mathcal{C}, \epsilon)$ and $\mathrm{L}^{\prime}=\left(\mathcal{P}^{\prime}, \mathcal{C}^{\prime}, \epsilon^{\prime}\right)$ be two Laguerre planes. If there exists a one to one and onto function $f$, which maps points and circles of $L$ to points and circles of $L^{\prime}$; respectively, and if,

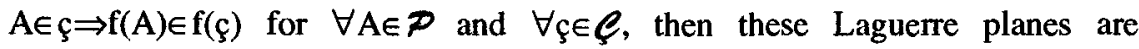
said to be isomorphic and such a function $f$ is said to be isomorphism.

If $L=L^{\prime}$ then the function $f$ is called an automorphism of the plane $\mathrm{L}$, [5]. The automorphism $\mathrm{f}$ maps parallel points to parallel points. Therefore an automorphism preserves the generators invariant in whole. Every automorphism $f$ maps a hyperbolic pencil to a hyperbolic pencil and a parabolic pencil to a parabolic pencil, that is,

$$
\begin{aligned}
f([A, B]) & =[f(A), f(B)] \\
f([A, \mathcal{G}]) & =[f(A), f(\mathcal{G})] .
\end{aligned}
$$

Restriction of an automorphism $f$ to the affine plane is an automorphism $\mathrm{fl}_{\mathrm{L}_{\mathrm{A}}}$ of $\mathrm{L}_{\mathrm{A}}$. Consider the affine plane $\mathrm{L}_{\mathrm{A}}$ as an embedding into Laguerre plane $L$. If the restriction $f_{L_{A}}$ acts on the points of the affine plane, then all points, which are parallel to $A$, are invariant under the mapping $f$.

Definition 2. An automorphism $f \neq I$ of a Laguerre plane is said to be Laguerre Inversion, if for all points $A \in \mathcal{P}$ with $A \neq f(A)$ it follows that $A$ and $f(A)$ are not parallel and all the circles passing through $A$ and $f(A)$ are invariant under $f$, where $I$ denotes the identity automorphism.

Proposition 1. Every Laguerre inversion is an involution.

Proof. Let $A \neq f(A)$, then $A \not f(A)$. Since the Laguerre inversion $f$ maps every circle of the hyperbolic pencil $[A, f(A)]$ into itself, the points $A, f(A)$ of all circles of the hyperbolic pencil $[A, f(A)]$ are invariant with respect to $f$. Then $A=f^{2}(A)$, that is $f$ is an involution.

Proposition 2. Let $A$ and $B$ are two different points of a Laguerre plane $L$ and $f$ is a Laguerre inversion. Then the set $\{A, f(A), B, f(B)\}$ is either concircular or a subset of union of two generators. 
Proof. Let $A$ denote a point which is not fix under $f$. If some points $B$ are not parallel to $A$ and $f(A)$, then there is a circle ç containing the points $A, f(A)$ and $B$. Since $\varsigma \in[A, f(A)], f(c)=\varsigma$ as according to the Definition 2. That is $f(B) \in €$ and $\{A, f(A), B, f(B)\}$ consists of the points of one circle. Now, let $B / / A$. Then $f(B) / / f(A)$. If $B / / f(A)$, then $f(B) / / f^{2}(A)=$ $A$, and by the Proposition 1 , the set $\{A, f(A), B, f(B)\}$ is the union of two different generators. Finally, if $A$ and $B$ are two invariant points of $f$, then obviously the set $\{A, B\}$ is a subset of the union of two generators.

Proposition 3. If a generator $\bar{A}$ is invariant under the Laguerre inversion $f$, then $\bar{A}$ is pointwise invariant under $f$.

Proof. Let $\bar{A}=f(\bar{A})$. Then every point $A$ of the generator $\bar{A}$ can be transformed to the point $\mathrm{f}(\mathrm{A})$, which is parallel to $\mathrm{A}$. According to the Definition 2 , $f$ does not change any points of the generator $\bar{A}$. In the case of $f(A)=A$ we have $\overline{f(A)}=\bar{A}=f(\bar{A})$. That is the generator $\bar{A}$ is pointwise invariant under $f$.

Proposition 4. The set of fixed points of a Laguerre inversion is empty or consists of the points of one or two generators.

Proof. Assume that a Laguerre inversion $f$ has the fixed point $A$. Then, according to Proposition $3, \bar{A}$ is pointwise invariant under $f$. If $B \notin \bar{A}$ is another fixed point of $f$, then the generators $\bar{A}$ and $\bar{B}(\bar{B} \neq \bar{A})$ are pointwise invariant under $f$. If $R \notin \bar{A} \cup \bar{B}$ and $f(R)=R$, then the automorphism $\left.f\right|_{L_{R}}$ preserves pointwise invariant two different lines of the affine plane $L_{R}$. Then $\left.f\right|_{L_{R}}=\left.I\right|_{L_{R}}$ and $f=I$. This is a contradiction to the condition of $\mathrm{f} \neq \mathrm{I}$.

Proposition 5. Let $f$ be a Laguerre inversion. The set of all circles, that are invariant under a Laguerre inversion $f$ and include a fixed point $A$, is a hyperbolic pencil when $\mathrm{f}(\mathrm{A}) \neq \mathrm{A}$ and a parabolic pencil when $\mathrm{f}(\mathrm{A})=\mathrm{A}$.

Proof. Let $\mathrm{f}(\mathrm{A}) \neq \mathrm{A}$. According to the Definition 2, the points $A$ and $f(A)$ are supports of the hyperbolic pencil $[A, f(A)]$, which are preserved under $f$. Inversely, if the circle $\mathcal{f}$ is invariant under $f$ and contains the point $A$, then for $A \in €$ we have $f(A) \in f(\xi)=\varsigma$, then $\varsigma$ is an element of hyperbolic pencil $[A, f(A)]$ and a fixed circle of $f$.

Now, let $f(A)=A$. In this case there will be two different situations. 
a) The generator $\bar{A}$ is unique generator which is pointwise invariant under $f$. If $B \notin \bar{A}$, then $B \neq f(B)$, that is, if $B H f(B)$, then $A, B$ and $f(B)$ are not pairwise parallel points. Denote by ç the circle which contains the points $A, B$ and $f(B)$ and define the following set

$$
\mathrm{K}:=\{\varsigma \in \mathcal{\varrho}: \mathcal{\xi}=(\operatorname{ABf}(\mathrm{B})), \forall \mathrm{B} \notin \overline{\mathrm{A}}\} .
$$

$K$ is the set of circles which are invariant under $f$ and covers the set $\mathcal{P} \bar{A}$. All circles of the set $K$ which $f(B) \neq B$ necessarily contains a point $(B, f(B))$. Suppose that there are two different circles $v$ and $w$ from $K$, which have another common point $D \neq A$, besides $A$. Since $v$ and $w$ are invariant under $f$ and $A$ is a fixed point of $f$, then $D$ is also a fixed point of $f$. But this contradicts to DHA. Therefore $K$ is a parabolic pencil of fix circles under $f$ with basepoint $A$.

b) f has two nonparallel fixed points $A$ and $D$. Let $B \notin \bar{A} \cup \bar{D}$. Then $\mathrm{BH} f(\mathrm{~B})$ and the circle $c=(\mathrm{ABf}(\mathrm{B}))$ is invariant under f. Let $S:=[A, \xi]$. Because of $f(A)=A$ and $c=f(c)$ the set $S$ is invariant in whole under f. Since each circle $w \in S$ contains the fixed point $\bar{D} \cap w$, then the set $S$ is elementwise invariant. In this case, A carries a parabolic pencil of fixed circles of $f$. Now let $w$ be a circle, which contains $A$ and invariant under f. Suppose that $[A, \xi]$ is a parabolic pencil of fixed circles with carrier point A. We have proved above the existence of such a pencil.

Suppose that $w \notin[A, c]$ and that $A \in w$, then $|w \cap v|=2$ for every $v \in[A, \xi\}]$ and $f(w \cap v)=w \cap v$. Therefore, both of elements of $w \cap v$ are also fixed points under $f$. Since this one is valid for every $v \in[A, c]$, then $w$ is pointwise invariant under $\mathrm{f}$. According to the Proposition 3 and 4 a Laguerre inversion can not possess three pairwise nonparallel fixed points. Therefore $w \in[A, \xi]$ and the Proposition 5 is proved.

\section{REFERENCES}

[1] BENZ, W., Pseudo-Ovale und Laguerre Ebenen. Abh. Math. Sem. Univ. Hamburg 27 (1964), 80-84.

[2] BENZ, W., MÄURER, H., Über die Grundlagen der Laguerre-Geonetrie. Jahresberg. Deutsch Math. Verein 67 (1964), 14-42.

[3] HARTMAN, E., Eine Klasse nicht einbettbarer reeller Laguerre-Ebenen. Journal of Geometry Vol. 13/1 (1979), 49-67. 
[4] KAYA, R., A Class of Ovoidal Laguerre Planes, Communications de la Faculté des Sciences de l'université d'Ankara. Série A1 Mathématiques, Tome 28 (1979), 1-5.

[5] KLEINEWILLINGHÖFER, R., Eine Klassifikation der Laguerre-Ebenen nach L-Streckungen und L-Translationen, Arch. Math. Vol. 34 (1980), 469-480. 